\title{
A SHORT PROOF OF THE COMPLETENESS OF THE LAGUERRE FUNCTIONS
}

LYNN H. LOOMIS

The Laguerre functions $\phi_{n}(x), n=0,1, \cdots$, can be defined ${ }^{1}$ as the coefficients of $t^{n}$ in the power series expansion about $t=0$ of the generating function

$$
F(x, t)=\exp \left(-\frac{x}{2}\left(\frac{1+t}{1-t}\right)\right) /(1-t) .
$$

TheOREM 1. The functions $\phi_{n}(x)$ form an orthonormal set over $(0, \infty)$.

The following proof is essentially well known. ${ }^{2}$ From the definition of $\phi_{n}(x)$ we see that $\int_{0}^{\infty} \phi_{m}(x) \phi_{n}(x) d x$ is the coefficient of $t^{m} s^{n}$ in the power series expansion about $(t, s)=(0,0)$ of

$$
\begin{aligned}
\int_{0}^{\infty} F(x, t) F(x, s) d x & =\int_{0}^{\infty}\left(\exp \left(-x \frac{1-t s}{(1-t)(1-s)}\right) /(1-t)(1-s)\right) d x \\
& =\frac{1}{1-t s}=\sum_{p=0}^{\infty}(t s)^{p} .
\end{aligned}
$$

Hence $\int_{0}^{\infty} \phi_{m}(x) \phi_{n}(x) d x$ is 1 or 0 according as $m=n$ or $m \neq n$, as was to be proved.

The interchange of the order of integration and summation can be justified by first making the interchange for the finite integral and then letting the upper limit approach infinity.

TheOREM 2. The functions $\phi_{n}(x)$ are complete (closed) in $L^{2}(0, \infty)$.

We use a special case of the well known theorem ${ }^{3}$ that if $f(z)$ $=a_{0}+a_{1} z+a_{2} z^{2}+\cdots$ is analytic and bounded in $|z|<1$ with boundary function $f\left(e^{i \theta}\right)$, then

$$
\int_{0}^{2 \pi}\left|f\left(e^{i \theta}\right)\right|^{2} d \theta=2 \pi \sum_{n=0}^{\infty}\left|a_{n}\right|^{2}
$$

Since step functions are dense in $L^{2}(0, \infty)$, it is sufficient, in order to prove the completeness of the $\phi_{n}(x)$, to prove that the closed linear

Received by the editors October 8, 1943.

${ }^{1}$ See Kaczmarz and Steinhaus, Theorie der Orthogonalreihen, p. 140, line 1, (55) and [482].

2 See, for example, G. Szegö, Orthogonal polynomials, Amer. Math. Soc. Colloquium Publications, vol. 23, p. 69.

${ }^{3}$ See Bieberbach, Lehrbuch der Funktionentheorie, vol. 1, p. 145. 
space which they determine includes the step functions, hence that it includes the characteristic function $g_{b}(x)$ of the interval $0 \leqq x \leqq b$. Thus if $a_{n}$ is the $n$th Laguerre coefficient of $g_{b}(x)$,

$$
a_{n}=\int_{0}^{\infty} g_{b}(x) \phi_{n}(x) d x=\int_{0}^{b} \phi_{n}(x) d x
$$

we have to prove that

$$
\int_{0}^{\infty}\left(g_{b}(x)-\sum_{n=0}^{N} a_{n} \phi_{n}(x)\right)^{2} d x \rightarrow 0
$$

as $N \rightarrow \infty$, that is, that

$$
b=\sum_{n=0}^{\infty}\left|a_{n}\right|^{2} .
$$

By (2) the Laguerre coefficients $a_{n}$ of $g_{b}(x)$ are the coefficients of $t^{n}$ in the expansion of

$$
\begin{aligned}
f(t) & =\int_{0}^{b}\left(\exp \left(-\frac{x}{2}\left(\frac{1+t}{1-t}\right)\right) /(1-t)\right) d x \\
& =-\frac{2}{1+t}\left[\exp \left(-\frac{b}{2}\left(\frac{1+t}{1-t}\right)\right)-1\right] .
\end{aligned}
$$

This function is analytic in $|t| \leqq 1$ except at $t=1$, and is bounded in $|t|<1$. Putting $t=e^{i \theta}$ we have

$$
f\left(e^{i \theta}\right)=-(1-i \tan (\theta / 2))\left(\exp \left(-\frac{b}{2} i \cot \frac{\theta}{2}\right)-1\right)
$$

and the substitutions $y=\tan (\theta / 2), x=b / 4 y$ give

(4) $\int_{0}^{2 \pi}\left|f\left(e^{i \theta}\right)\right|^{2} d \theta=8 \int_{-\infty}^{\infty} \sin ^{2}(b / 4 y) d y=2 b \int_{-\infty}^{\infty} \frac{\sin ^{2} x}{x^{2}} d x=2 \pi b$.

The desired result (3) follows from (1) and (4).

HARVARD UNIVERSITY 